\title{
Spin-wave coupling to electromagnetic cavity fields in dysposium ferrite
}

\author{
M. Białek $\odot,{ }^{*}$ A. Magrez, and J.-Ph. Ansermet \\ Institute of Physics, École Polytechnique Fédérale de Lausanne (EPFL), 1015 Lausanne, Switzerland
}

(Received 10 April 2019; revised manuscript received 10 December 2019; published 9 January 2020)

\begin{abstract}
Coupling of spin waves with electromagnetic cavity field is demonstrated in an antiferromagnet, dysprosium ferrite $\left(\mathrm{DyFeO}_{3}\right)$. By measuring transmission at $0.2-0.35 \mathrm{THz}$ and sweeping sample temperature, magnonphoton coupling signatures were found at crossings of spin-wave resonances with Fabry-Pérot cavity modes formed in samples. The obtained spectra are explained in terms of classical electrodynamics and a microscopic model.
\end{abstract}

DOI: 10.1103/PhysRevB.101.024405

\section{INTRODUCTION}

Coupling of matter and electromagnetic radiation [1] is a topic of great interest in solid state physics research because of their hybrid quantum nature [2]. In the terahertz range, phonon-polaritons are a well-known example of a lightmatter coupling. Recently, polaritons in the terahertz region were shown with intersubband transitions [3,4], cyclotron resonance [5], and plasmons [6] in two-dimensional electron gases, as well as with intermolecular transitions in organic materials [7]. In these systems, a strong-coupling regime can be achieved when losses are smaller than the exchange rate between light and matter [8], giving rise to the vacuum Rabi splitting. Polaritons are composite particles, which are studied in basic research on quantum optics [2], and can be considered for use in quantum computing and quantum memories [9-13]. The coupling of electromagnetic cavity modes to magnons was researched intensively in ferromagnets at gigahertz frequencies $[12,14,15]$, meeting with expectations of energyefficient spintronic devices [16]. Coupling of magnons with superconducting qubits was also investigated $[17,18]$. The Purcell enhancement and the vacuum Rabi splitting were demonstrated in ferromagnetic materials [19-22].

It is interesting to investigate magnon-photon coupling in antiferromagnetic materials [1] in view of their highfrequency spin dynamics [23-27], comparing to that of ferromagnets. As this phenomenon is readily taken into account by classical electrodynamics, it was accounted for in the analysis of optical investigations of antiferromagnetic materials, for instance in Refs. [28-32]. Some experimental reports focused on characterization of interaction of antiferromagnetic magnons with photons in $\mathrm{FeF}_{2}$ [33,34], $\mathrm{NiO}$ [35], $\mathrm{TmFeO}_{3}$ [36], and in $\mathrm{ErFeO}_{3}$ [37,38]. Most of these reports use classical electrodynamics. A microscopic picture was developed

\footnotetext{
*marcin.bialek@epfl.ch
}

Published by the American Physical Society under the terms of the Creative Commons Attribution 4.0 International license. Further distribution of this work must maintain attribution to the author(s) and the published article's title, journal citation, and DOI. in Ref. [37]. Here, we confront the classical electrodynamic model with predictions of a microscopic model by estimating, quantitatively from our data, the strength of the interaction of antiferromagnetic spin waves with electromagnetic cavity modes in dysprosium ferrite $\mathrm{DyFeO}_{3}$ (DFO).

Dysprosium ferrite is an orthogonally distorted perovskite. It shows antiferromagnetic ordering below a Néel temperature of about $640 \mathrm{~K}$ [39]. Dzyaloshinskii-Moriya leads to a weak ferromagnetism. In DFO, the spin canting allows two antiferromagnetic resonance modes to be excited: the quasiferromagnetic (qFMR) and the quasiantiferromagnetic (qAFMR) [28,29,39-41], which are excited by the magnetic component of radiation [42].

\section{EXPERIMENTAL}

Our measurements were performed above $400 \mathrm{~K}$, where both resonances monotonously soften with rising temperature. We used polycrystalline samples for their isotropic properties, as large anisotropic properties in crystals might hinder the coupling [43]. We have created disk-shaped samples of 1.0 and $0.6 \mathrm{~mm}$ thicknesses, thus having different cavitymode spectra. A sample was placed in a furnace, which allowed one to control temperature up to $700 \mathrm{~K}$. By using quasioptical methods, we measured transmission with our continuous-wave terahertz spectrometer based on frequency extenders to a vector network analyzer [44]. This complex signal $S_{21}(f, T, H)$ is a function of frequency $f$, temperature $T$, and magnetic field $H$. We report its power amplitude in decibel units and its phase in degrees. In order to extract a signal related to magnetic resonances, we measured transmission through a sample at different temperatures $T$. We obtained temperature-differential spectra by subtracting averaged spectra measured at subsequent temperatures:

$$
\frac{\partial S_{21}}{\partial T}=\Delta T^{-1}\left[S_{21}(f, T+\Delta T, 0)-S_{21}(f, T, 0)\right]
$$

with $\Delta T=1 \mathrm{~K}$. We used this technique to measure spinwave resonances in bismuth ferrite at high [45] and low temperatures [43]. 


\section{MODEL}

In classical electrodynamics, the dispersion of electromagnetic radiation in a material with a resonance is modified. This leads to the creation of two polariton states split by a frequency representing coupling of the resonance with the electromagnetic radiation [1]. In DFO, we model magnetic susceptibility $\mu_{c}(f, T, H)$ using Lorentzian distributions

$$
\mu_{c}(f, T, H)=1+\sum_{m=1}^{M} \frac{\Delta \mu_{m}(T, H) f_{m}^{2}(T, H)}{f_{m}^{2}(T, H)-f^{2}-i f \gamma_{m}(T, H)},
$$

where, for the $m$ th magnetic resonance, $f_{m}$ is its frequency, $\gamma_{m}$ its width, and $\Delta \mu_{m}$ is its input to the zero-frequency magnetic susceptibility. In the case of DFO, $M=2$, with $m=1$ corresponding to the qFMR and $m=2$ to the qAFMR.

Ferrites have dielectric functions $\epsilon_{c}(f, T)$, which, in our experimental frequency range, i.e., far from phonon resonant frequencies, can be approximated as linear around a convenient point $\left(T_{0}=400 \mathrm{~K}, f_{0}=0.3 \mathrm{THz}\right)$

$$
\epsilon_{c}(f, T)=\epsilon_{00}+a\left(f-f_{0}\right)+b\left(T-T_{0}\right) .
$$

For our polycrystalline samples, we assume an effective [46,47] magnetic susceptibility as $\sqrt{\mu}=p \sqrt{\mu_{c}}+(1-p)$ and effective dielectric function as $\sqrt{\epsilon}=p \sqrt{\epsilon_{c}}+(1-p)$, where the factor $p=0.64$ is a volume fraction of material to air in our pelletized samples [43]. This value is the maximum density of random-packed hard spheres [48]. This assumption allows us to obtain values of $\epsilon_{c}$ and $\mu_{c}$ that are comparable with literature values for single-crystal samples [29,49]. The complex wave vector is $k=2 \pi f \sqrt{\epsilon \mu} / c$, where $c$ is the speed of light in vacuum. Transmission of electric field $t(f, T, H)$ though a slab of a thickness $d$ and infinite lateral dimensions is [50]

$$
t(f, T, H)=\frac{\left(1-r^{2}\right) e^{i k d}}{1-r^{2} e^{i 2 k d}}
$$

where $r^{2}=(\sqrt{\epsilon}-\sqrt{\mu})^{2} /(\sqrt{\epsilon}+\sqrt{\mu})^{2}$ is the square of the reflection coefficient at the vacuum-material interface. For frequencies far away from resonances, Eq. (4) implies an interference pattern, related to subsequent cavity modes of a slab, called Fabry-Pérot modes. At $300 \mathrm{GHz}$, in our polycrystalline DFO samples with the refractive index $\sqrt{\epsilon \mu} \approx 3.6$, the wavelength is about $300 \mu \mathrm{m}$. With rising temperature, $\sqrt{\epsilon \mu}$ increases, which shortens the period of the interference pattern. The magnetic susceptibility takes into account the effect of the magnetic resonance. When its frequency is close to a mode of a cavity, both the magnon and the cavity lines are altered by the matter-photon interaction. Since the magnetic resonance frequencies have a much stronger temperature dependence than that of the interference pattern, they cross several interference pattern minima as temperature rises. We calculated

$$
\left\|\frac{\partial S_{21}}{\partial T}\right\|=\Delta T^{-1} 20 \log _{10}\left\|\frac{t(f, T+\Delta T, 0)}{t(f, T, 0)}\right\|
$$

to fit amplitude of temperature-differential spectra, where $\Delta T=1 \mathrm{~K}$ is the temperature step. We calculated phase of temperature-differential spectra using

$$
\arg \left(\partial S_{21} / \partial T\right)=\arg [t(f, T+\Delta T, 0)-t(f, T, 0)] .
$$

\section{RESULTS}

The experimental temperature-differential signal amplitude for the 1.0-mm-thick DFO sample is shown in Fig. 1(a). These data show clearly that the resonant lines are distorted when they cross the sequence of sample-cavity modes. The amplitude and the widths of the resonances are altered because of their interaction with the electromagnetic standing waves. This is accounted for by Eq. (5) as shown in Fig. 1(c). Thus, we find that this model reproduces most of the important features of Fig. 1(a). The fitting parameters are parameters of the simplified dielectric function [Eq. (3)] and of the magnetic susceptibility [Eq. (2)]. We assumed that resonance frequencies have a temperature dependence described by a power law $f_{m}^{*}\left(1-T / T_{N}\right)^{\beta_{m}}$, applicable when approaching the Néel temperature $T_{N}$ [51], where $f_{m}^{*}$ has a unit of frequency and $\beta_{m} \approx \frac{1}{3}$ in the case of DFO. To improve the quality of our fits, we assumed that magnetic resonances have linear dependences of their widths and amplitudes on temperature. These formulas and values of fit parameters are given in the Supplemental Material [52].

We measured the temperature-differential phase of transmitted electric field, as presented in Fig. 1(b). Despite high noise, features predicted in Fig. 1(d) are observed in the experiment. The phase clearly reveals interactions between electromagnetic waves and magnetization dynamics. In Fig. 2(a), a spectrum obtained at a temperature between crossing points shows that the qAFMR can be accounted for with a harmonic model. In Fig. 2(b), the spectrum obtained at the temperature of the crossing with the $l=6$ cavity mode shows a structure that cannot be explained using a single oscillator. It is due to two polariton states which are not well separated, i.e., they are in a weak-coupling regime [2]. We used this phase prediction to estimate the cavity mode-magnetic resonance coupling strength. Thus, we superimposed on Fig. 1(d) predictions of the harmonic coupling model $[1,12,53]$

$$
f_{ \pm}=\frac{1}{2}\left(f_{(l)}+f_{m} \pm \sqrt{\left(f_{(l)}-f_{m}\right)^{2}+4 \kappa^{2} f_{(l)}}\right),
$$

where $f_{ \pm}$indicates upper and lower polariton frequencies, $f_{(l)}$ is the $l$ th cavity mode frequency, and $f_{m}$ with $m=1,2$ are the resonance frequencies. The coupling strength is given by [12]

$$
\kappa=\frac{g_{s} \mu_{B}}{2 h} \sqrt{\frac{\mu_{0} h}{2} p \rho}
$$

where $g_{s}=2, \rho$ is density of resonators, and $p=0.64$ is the mass filling factor of our polycrystalline sample $[43,48]$. Equation (8) assumes that a magnon is coupled to a single electromagnetic cavity mode with a coupling strength [54] $g_{s} \mu_{B} B_{0} / 2 h$, where $B_{0}=\sqrt{\mu_{0} h f_{(l)} / 2 V_{(l)}}$ is the magnetic component of vacuum fluctuations [55]. The amplitude of these is only $\approx 5 \times 10^{-11} \mathrm{~T}$ for an electromagnetic mode at $0.3 \mathrm{THz}$ taking the volume $V_{(l)} \approx 16 \pi \times 10^{-9} \mathrm{~m}^{3}$ of the 1 -mm-thick sample. This is about three orders of magnitude smaller than the amplitude of the terahertz field in our experiment. However, in an ensemble of $N$ resonators that collectively interact with a cavity mode, coupling is increased by a factor 

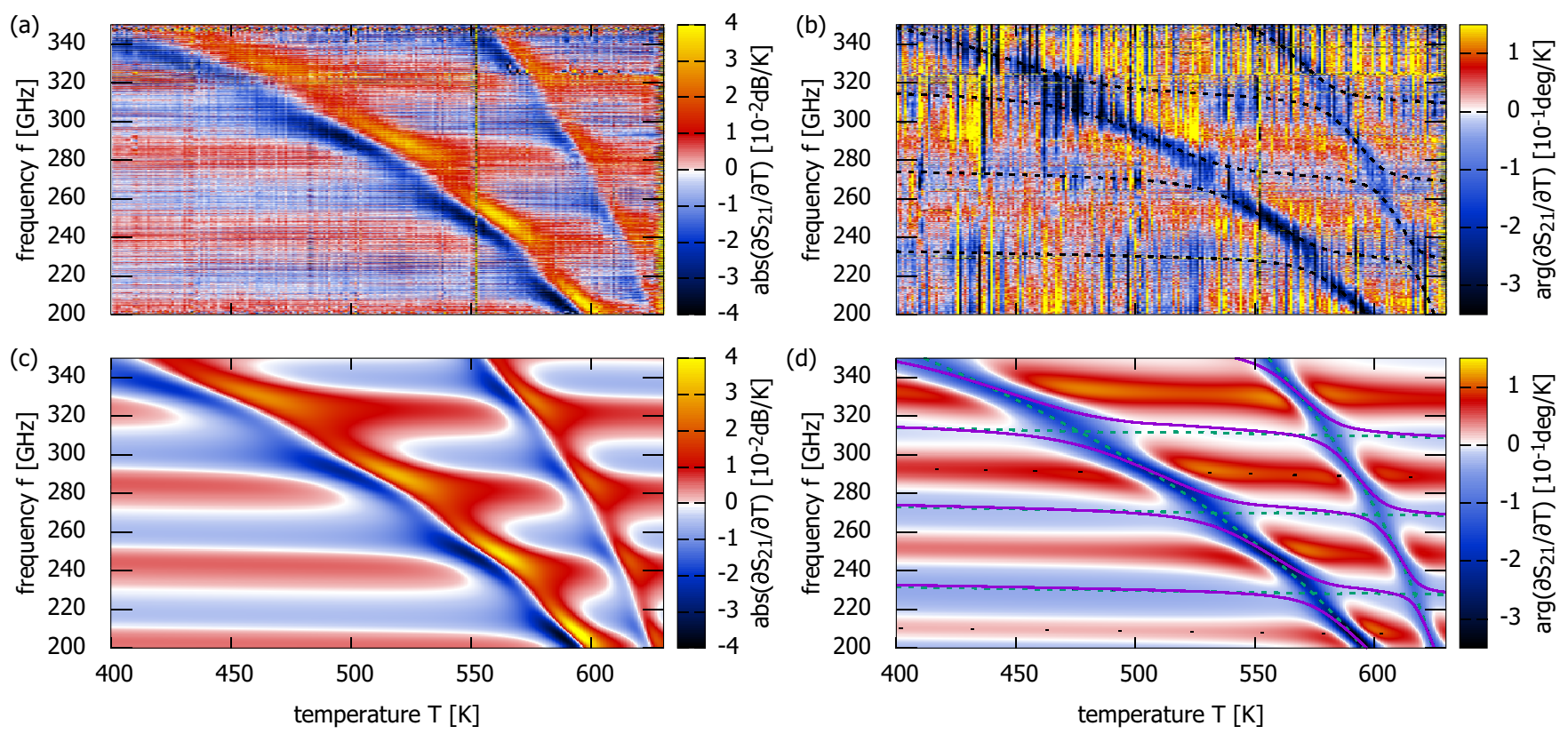

FIG. 1. Experimental temperature-differential spectra for the 1.0-mm-thick DFO sample: (a) amplitude; (b) median-shifted phase with dashed black lines showing coupled modes. Fit of the model to the amplitude data: (c) magnitude; (d) phase. In the segment (d), green dashed lines show uncoupled spin-wave and cavity modes and purple lines show coupled modes.

$\sqrt{N}[12,37,53,54,56]$. Thus, the collective coupling strength $\kappa$ depends only on oscillator density $\rho=N / V_{c}$ and the ratio $p$ of a crystalline volume $V_{c}$ to a cavity volume $V_{(l)}[12,37]$. We take $\rho=\frac{1}{3} \rho_{\mathrm{Fe}}$, where $\rho_{\mathrm{Fe}}=1.76 \times 10^{28} \mathrm{~m}^{-3}$ is density of iron atoms in DFO [57]. This factor reflects the fact that in a polycrystalline material, on average, only $1 / 3$ of magnetic dipoles are excited by linear polarized electromagnetic wave. Under this assumption $\kappa=1.78 \times 10^{4} \mathrm{~Hz}^{1 / 2}$, which results

(a)

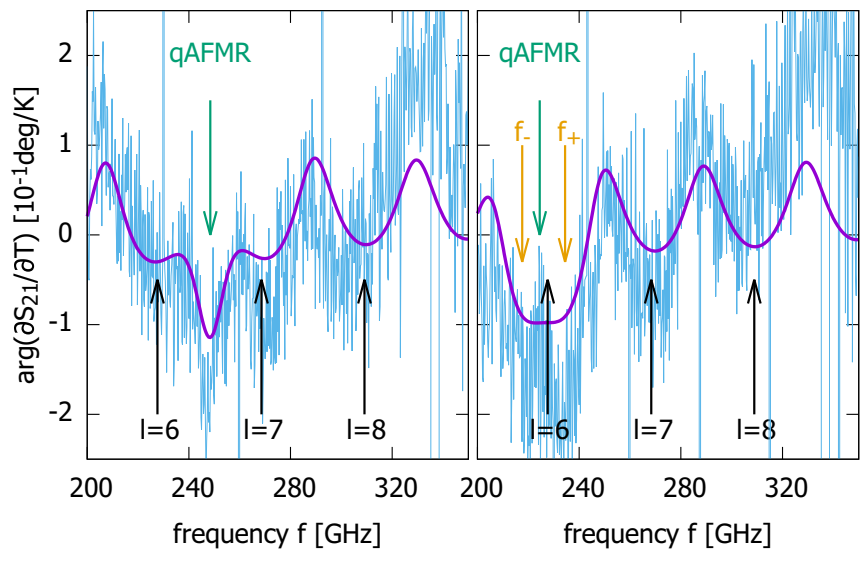

FIG. 2. Examples of phase spectra extracted from Fig. 1(b). (a) Spectrum at a temperature not showing light-matter interaction; (b) temperature close to a crossing point. Arrows mark positions of cavity modes, qAFMR, and upper and lower polaritons. The violet lines show fits obtained using data in the entire temperature range [Fig. 1(d)], thus better reflecting average properties of the sample. in a splitting $2 \kappa \sqrt{f_{(l)}} \approx 19.5 \mathrm{GHz}$ with a cavity mode of $f_{(l)}=0.3 \mathrm{THz}$.

Using Eq. (7), we calculated modes undergoing subsequent interactions with the same coupling strength $\kappa$. We determined $f_{(l)}$ from the condition $c(l-1 / 2)=2 \operatorname{Re}(\sqrt{\epsilon \mu}) f_{(l)} d$ for a minimum of $\arg \left(\partial S_{21} / \partial T\right)$. In the case of the 1-mm-thick sample, the lowest visible mode at $\approx 230 \mathrm{GHz}$ has $l=6$. Resonant modes frequencies were obtained from the same fit to the experimental $\partial S_{21} / \partial T$ magnitude. For the $1.0-\mathrm{mm}$ thick sample, the temperature dependence of both coupled and uncoupled modes is presented in Fig. 1(d).

\section{SUMMARY}

In summary, we have observed coupling of spin waves with electromagnetic fields in high-temperature antiferromagnet $\mathrm{DyFeO}_{3}$. We observed the coupling of terahertz-frequency magnetic resonances to modes of cavities formed by the samples themselves. Our research demonstrates a possibility for a spin-cavitronic system based on antiferrromagnetic resonances. To account for our results, we applied both classical electrodynamics and a microscopic magnon-photon coupling model [12], taking into account the polycrystalline nature of our samples.

\section{ACKNOWLEDGMENTS}

We would like to thank Prof. C.-M. Hu of University of Manitoba for very fruitful discussions and Claude Amendola for help in the samples preparation. This work was partially supported by a Requip (206021150707/1) grant of the Swiss National Science Foundation and by EPFL. 
[1] D. L. Mills and E. Burstein, Rep. Prog. Phys. 37, 817 (1974).

[2] G. Khitrova, H. M. Gibbs, M. Kira, S. W. Koch, and A. Scherer, Nat. Phys. 2, 81 (2006).

[3] A. A. Anappara, S. De Liberato, A. Tredicucci, C. Ciuti, G. Biasiol, L. Sorba, and F. Beltram, Phys. Rev. B 79, 201303(R) (2009).

[4] Y. Todorov, A. M. Andrews, R. Colombelli, S. De Liberato, C. Ciuti, P. Klang, G. Strasser, and C. Sirtori, Phys. Rev. Lett. 105, 196402 (2010).

[5] A. Bayer, M. Pozimski, S. Schambeck, D. Schuh, R. Huber, D. Bougeard, and C. Lange, Nano Lett. 17, 6340 (2017).

[6] Y. Yu, Z. Zheng, H. Qin, J. Sun, Y. Huang, X. Li, Z. Zhang, D. Wu, Y. Cai, B. Zhang, and V. V. Popov, Opt. Express 26, 31794 (2018).

[7] R. Damari, O. Weinberg, D. Krotkov, N. Demina, K. Akulov, A. Golombek, T. Schwartz, and S. Fleischer, Nat. Commun. 10, 3248 (2019).

[8] G. L. Paravicini-Bagliani, G. Scalari, F. Valmorra, J. Keller, C. Maissen, M. Beck, and J. Faist, Phys. Rev. B 95, 205304 (2017).

[9] J. H. Wesenberg, A. Ardavan, G. A. D. Briggs, J. J. L. Morton, R. J. Schoelkopf, D. I. Schuster, and K. Mølmer, Phys. Rev. Lett. 103, 070502 (2009).

[10] D. I. Schuster, A. P. Sears, E. Ginossar, L. DiCarlo, L. Frunzio, J. J. L. Morton, H. Wu, G. A. D. Briggs, B. B. Buckley, D. D. Awschalom, and R. J. Schoelkopf, Phys. Rev. Lett. 105, 140501 (2010).

[11] Y. Kubo, F. R. Ong, P. Bertet, D. Vion, V. Jacques, D. Zheng, A. Dréau, J.-F. Roch, A. Auffeves, F. Jelezko, J. Wrachtrup, M. F. Barthe, P. Bergonzo, and D. Esteve, Phys. Rev. Lett. 105, 140502 (2010).

[12] H. Huebl, C. W. Zollitsch, J. Lotze, F. Hocke, M. Greifenstein, A. Marx, R. Gross, and S. T. B. Goennenwein, Phys. Rev. Lett. 111, 127003 (2013).

[13] A. Frisk Kockum, A. Miranowicz, S. De Liberato, S. Savasta, and F. Nori, Nat. Rev. Phys. 1, 19 (2019).

[14] B. M. Yao, Y. S. Gui, Y. Xiao, H. Guo, X. S. Chen, W. Lu, C. L. Chien, and C.-M. Hu, Phys. Rev. B 92, 184407 (2015).

[15] V. Castel, R. Jeunehomme, J. Ben Youssef, N. Vukadinovic, A. Manchec, F. K. Dejene, and G. E. W. Bauer, Phys. Rev. B 96, 064407 (2017).

[16] F. Matsukura, Y. Tokura, and H. Ohno, Nat. Nanotechnol. 10, 209 (2015).

[17] Y. Tabuchi, S. Ishino, A. Noguchi, T. Ishikawa, R. Yamazaki, K. Usami, and Y. Nakamura, Science 349, 405 (2015).

[18] D. Lachance-Quirion, Y. Tabuchi, S. Ishino, A. Noguchi, T. Ishikawa, R. Yamazaki, and Y. Nakamura, Sci. Adv. 3, e1603150 (2017).

[19] X. Zhang, C.-L. Zou, L. Jiang, and H. X. Tang, Phys. Rev. Lett. 113, 156401 (2014).

[20] Y. Tabuchi, S. Ishino, T. Ishikawa, R. Yamazaki, K. Usami, and Y. Nakamura, Phys. Rev. Lett. 113, 083603 (2014).

[21] L. Bai, M. Harder, Y. P. Chen, X. Fan, J. Q. Xiao, and C.-M. Hu, Phys. Rev. Lett. 114, 227201 (2015).

[22] H. Maier-Flaig, M. Harder, R. Gross, H. Huebl, and S. T. B. Goennenwein, Phys. Rev. B 94, 054433 (2016).

[23] T. Kampfrath, A. Sell, G. Klatt, A. Pashkin, S. Mährlein, T. Dekorsy, M. Wolf, M. Fiebig, A. Leitenstorfer, and R. Huber, Nat. Photonics 5, 31 (2011).

[24] T. Jungwirth, X. Marti, P. Wadley, and J. Wunderlich, Nat. Nanotechnol. 11, 231 (2016).
[25] S. Baierl, M. Hohenleutner, T. Kampfrath, A. K. Zvezdin, A. V. Kimel, R. Huber, and R. V. Mikhaylovskiy, Nat. Photonics 10, 715 (2016).

[26] P. Nemec, M. Fiebig, T. Kampfrath, and A. V. Kimel, Nat. Phys. 14, 229 (2018).

[27] S. Schlauderer, C. Lange, S. Baierl, T. Ebnet, C. P. Schmid, D. C. Valovcin, A. K. Zvezdin, A. V. Kimel, R. V. Mikhaylovskiy, and R. Huber, Nature (London) 569, 383 (2019).

[28] A. M. Balbashov, A. A. Volkov, S. P. Lebedev, A. A. Mukhin, and A. S. Prokhorov, J. Exp. Theor. Phys. 61, 543 (1985).

[29] G. V. Kozlov, S. P. Lebedev, A. A. Mukhin, A. S. Prokhorov, I. V. Fedorov, A. M. Balbashov, and I. Y. Parsegov, IEEE Trans. Magn. 29, 3443 (1993).

[30] R. V. Mikhaylovskiy, E. Hendry, V. V. Kruglyak, R. V. Pisarev, T. Rasing, and A. V. Kimel, Phys. Rev. B 90, 184405 (2014).

[31] R. V. Mikhaylovskiy, E. Hendry, A. Secchi, J. H. Mentink, M. Eckstein, A. Wu, R. V. Pisarev, V. V. Kruglyak, M. I. Katsnelson, T. Rasing, and A. V. Kimel, Nat. Commun. 6, 8190 (2015).

[32] R. V. Mikhaylovskiy, T. J. Huisman, A. I. Popov, A. K. Zvezdin, T. Rasing, R. V. Pisarev, and A. V. Kimel, Phys. Rev. B 92, 094437 (2015).

[33] R. Sanders, V. Jaccarino, and S. Rezende, Solid State Commun. 28, 907 (1978).

[34] M. R. F. Jensen, S. A. Feiven, T. J. Parker, and R. E. Camley, J. Phys.: Condens. Matter 9, 7233 (1997).

[35] J. Li, T. Higuchi, N. Kanda, K. Konishi, S. G. Tikhodeev, and M. Kuwata-Gonokami, Opt. Express 19, 22550 (2011).

[36] K. Grishunin, T. Huisman, G. Li, E. Mishina, T. Rasing, A. V. Kimel, K. Zhang, Z. Jin, S. Cao, W. Ren, G.-H. Ma, and R. V. Mikhaylovskiy, ACS Photonics 5, 1375 (2018).

[37] X. Li, M. Bamba, N. Yuan, Q. Zhang, Y. Zhao, M. Xiang, K. $\mathrm{Xu}$, Z. Jin, W. Ren, G. Ma, S. Cao, D. Turchinovich, and J. Kono, Science 361, 794 (2018).

[38] P. Sivarajah, A. Steinbacher, B. Dastrup, J. Lu, M. Xiang, W. Ren, S. Kamba, S. Cao, and K. A. Nelson, J. Appl. Phys. 125, 213103 (2019).

[39] R. M. White, R. J. Nemanich, and C. Herring, Phys. Rev. B 25, 1822 (1982).

[40] A. K. Zvezdin and V. M. Matveev, J. Exp. Theor. Phys. 77, 1076 (1979) [Sov. Phys. JETP 50, 543 (1979)].

[41] N. Koshizuka and K. Hayashi, J. Phys. Soc. Jpn. 57, 4418 (1988).

[42] A. H. M. Reid, T. Rasing, R. V. Pisarev, H. A. Dürr, and M. C. Hoffmann, Appl. Phys. Lett. 106, 082403 (2015).

[43] M. Białek, T. Ito, H. Rønnow, and J.-P. Ansermet, Phys. Rev. B 99, 064429 (2019).

[44] C. Caspers, V. P. Gandhi, A. Magrez, E. de Rijk, and J.-P. Ansermet, Appl. Phys. Lett. 108, 241109 (2016).

[45] M. Białek, A. Magrez, A. Murk, and J.-P. Ansermet, Phys. Rev. B 97, 054410 (2018)

[46] J. R. Birchak, C. G. Gardner, J. E. Hipp, and J. M. Victor, Proc. IEEE 62, 93 (1974).

[47] W. Heller, Phys. Rev. 68, 5 (1945).

[48] C. Song, P. Wang, and H. A. Makse, Nature (London) 453, 629 (2008).

[49] R. P. S. M. Lobo, R. L. Moreira, D. Lebeugle, and D. Colson, Phys. Rev. B 76, 172105 (2007). 
[50] Principles of Optics, 6th ed., edited by M. Born and E. Wolf (Pergamon, New York, 1980), pp. 1-70.

[51] M. Eibschütz, S. Shtrikman, and D. Treves, Solid State Commun. 4, 141 (1966).

[52] See Supplemental Material at http://link.aps.org/supplemental/ 10.1103/PhysRevB.101.024405 for experimental details and fit parameters of the electromagnetic model.

[53] M. Harder, L. Bai, C. Match, J. Sirker, and C. Hu, Sci. China Phys., Mech. Astron. 59, 117511 (2016).
[54] O. O. Soykal and M. E. Flatté, Phys. Rev. Lett. 104, 077202 (2010).

[55] T. Niemczyk, F. Deppe, M. Mariantoni, E. P. Menzel, E. Hoffmann, G. Wild, L. Eggenstein, A. Marx, and R. Gross, Supercond. Sci. Technol. 22, 034009 (2009).

[56] M. G. Raizen, R. J. Thompson, R. J. Brecha, H. J. Kimble, and H. J. Carmichael, Phys. Rev. Lett. 63, 240 (1989).

[57] M. Eibschütz, Acta Crystallogr. 19, 337 (1965). 